\title{
About some approaches to problem of metals and alloys microstructures classification based on neural network technologies
}

\author{
R. Klestov \\ Department of Computational Mathematics and Mechanics \\ Perm National Research Polytechnic University \\ Perm, Russia \\ klestovroman@gmail.com
}

\author{
A. Klyuev \\ Department of Computational Mathematics and Mechanics \\ Perm National Research Polytechnic University \\ Perm, Russia \\ kav@gelicon.biz
}

\author{
V. Stolbov \\ Department of Computational Mathematics and Mechanics \\ Perm National Research Polytechnic University \\ Perm, Russia \\ valeriy.stolbov@gmail.com
}

\begin{abstract}
Two approaches to the decision of the problem of of metal alloys microstructures classification using neural network technologies are considered. Characteristics of the existing methods of recognition of grains circuits and phases of difficult microstructures are selected and described. A certain sequence of application of methods of the segmentation problem decision and classification by means of the developed neuronets is offered. Two alternative candidate solutions of the classification problem depending on adequate accuracy and volume of a set of input data are offered. Learning database for neural net with providing examples that show differences of same picture on various scales is described. The mathematical feature of operation of each of methods is described. Examples of architecture of a neuronet and samples of input data are given.
\end{abstract}

Keywords-functional materials; strength properties; complex estimation; grain-phase structure; problem of segmentation; classification of microstructures; neural network technologies

\section{INTRODUCTION}

Last years the role of the so-called metal "functional materials" differing in the given complex of utilization properties (durability, conductance, thermal stability, etc.) created due to use of new technologies of manufacturing or special technologies of its later physicomechanical processing and, as a result, the created microstructure [1,2] sharply increased in technique. At the same time, necessity of development of new means of the automated analysis of complex grain-phase structures on meso- or the microscale levels allowing not only to recognize and classify complex structures, but also to define numerical values of their key parameters increases. The problem of the microstructure analysis of materials based on pictures of the microsections and images received by means of atomic and force microscopy or other tools is very relevant as its decision carries to development of automated methods of quality control of the created materials $[3,4]$. It, in turn, promotes development of new perspective methods of manufacture of the functional materials and forecasting methods of their utilization properties. The important particular problem of prediction of material characteristics at the stage of its development is the problem of microstructures classification by a set of the preset numerical values of its macroproperties. This problem belongs to intellectual problems [5,6] which decision requires development of the new methods based on theories of an artificial intelligence [7]. In this operation two possible approaches to the decision of the considered problem of classification based on neural network technologies [7] are offered.

\section{PROBLEM DEFINITION}

Consider the problem of microstructures classification of the metal functional materials (FM). The problem here is understood as referring of the researched microstructure to this or that class depending on the given set of values of physic mechanical characteristics of material. The class is understood as the material which numerical values of all characteristics are situated in the given intervals.

It is considered that several digital images of microsection of the increase researched by FM at different scales are this. It is required to define to what of the given classes on a set of physic mechanical characteristics belongs researched by FM.

For resolving this problem, it is necessary to have already labeled database of microstructure's pictures with their physical-mechanical properties. The image marking is understood as application to it the additional information obtained by the expert-metallurgist about parameters of the figured microstructure (grain point, a volume fraction of phases, etc.). It is necessary to point out this information is not 
full that complicates application of standard methods of classification and requires development of new technologies.

Therefore, for recognition of the image and the decision of the problem of classification it is offered to use the neural network technologies, which showed the efficiency in case of the decision of similar intellectual problems [8].

\section{ONE-STAGE APPROACH}

One-stage approach consists in the direct decision of the problem of classification without additional methods of processing and correction of areas of interest for a neural network. In this operation the convolution neural network (Convolutional Neural Network, CNN) which represents the special architecture of the artificial neural networks (ANN) for the first time offered by Yann LeCun $[9,10]$ and initially intended for effective image understanding is used. Today, deep artificial neural networks are applied in many areas, for example, to search of the user of a social network in his photo or to formation of recommendations of purchase of goods and many other things [11, 12]. Classification of images remains classical and most popular option of application.

Classification of the image is that on an input of $\mathrm{CNN}$ is given the material image, and on an output of a network the result in the form of a class turns out or group of the classes characterizing a central object or objects which are figured on it. The convolution network recognizes the transferred image which appears as a set of pixels, irrespective of quantity and layout of objects as they are seen by a human eye. Depending on image size and its color format the array can be a miscellaneous, we will allow $256 \times 256 \times 3$ where 3 - the number of RGB channels. The value from 0 to 255 which describes intensity of pixel in the channel is appropriated to each of these pixels or array cells. Objects on the image can be located differently, besides, they can is as on front, and a background, layout not entirely in a frame is also possible, they can shield partially with each other, etc. Recognition accuracy of neural network depends on such features as layout of objects. As a rule, the accuracy of recognition is characterized by value of confidence of a network that on the image there is an object of a certain class. Value of confidence changes in range from 0 to 1 . For an object of one class it can be 0.7 , for another 0.9 . Thus, the main idea of recognition consists in transmission to an input of a network of a set of values of pixels and receiving on an output of a set of values of confidence for all classes of objects which can be recognized on this image. Selecting from this set elements with the maximum confidence it is possible to draw conclusions about existence of these or those objects in the field of the interest of a network.

In case of the decision of the considered problem by means of such approach use of the database which consists of pictures of microsections, whenever possible in different increases, and the table which is attached to images containing data on physic mechanical characteristics of the metal provided on the image will be required.

The algorithm of the decision of the problem of microstructure image classification researched by FM by means of a neural network is given in Fig. 1.



Fig 1. Algorithm of classification of image using CNN

Feature of the offered algorithm is simplicity of structure of a neural network for implementation and absence of need of finishing of source images for the qualifier. In other words, in case of such approach at a grade level on an input of neural network, images of microsection are provided in Fig. 2, and the known physical-mechanical properties of the figured microsection are described in the database move.

The neural network sets compliance between contents of the image and its properties and thus creates statuses of neurons. In case of the trained qualifier, by transmission to an image input to the neuronet working in the recognition mode she will try to pick up the template, next to this image, and to 
select the predicted physical-mechanical characteristics of metal for a level of likeness.

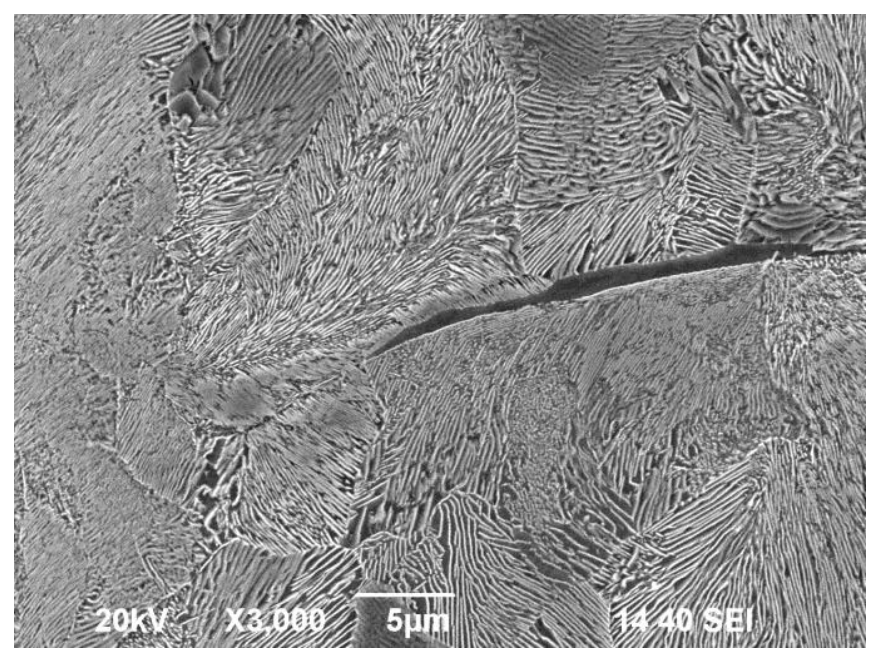

Fig. 2. Example of the image of a steel microstructure from the database

In Fig. 2 the image of microsection in case of increase in $\mathrm{X} 3000$ on which it is possible to define a row of characteristics, for example, interlamellar distance is provided. For class definition of the image the neuronet, which architecture is provided in Fig. 3, will be used.

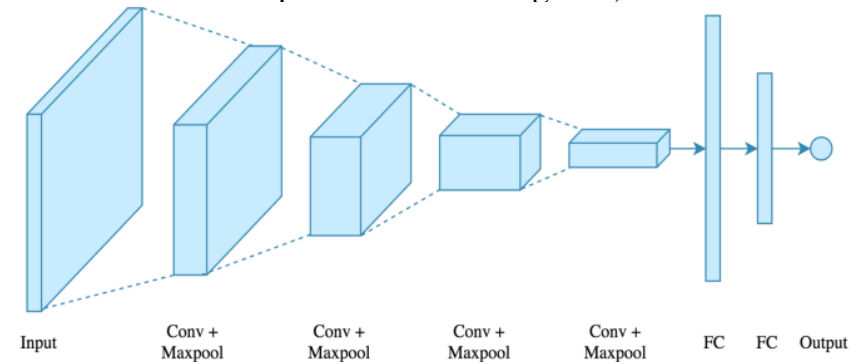

Fig. 3. Diagram of architecture of a neural network.

The input layer of a network serves for image transfer in a network. The specified size 1280x960x3 of an input layer shows that 3686400 pixels where is $1280 \times 960$ an image size, and 3 - the number of channels of representation of color (RGB, value range from 0 to 255 ) will be involved $1228800 * 3$ $=3686400$.

Further, the considered network contains two layers of a convolution, three layers of subselection and a final fullmeshed layer. This layer is necessary to support the final phase of classification, i.e. it provides a signal, a certain level, on qualifier outputs after the previous layers selected all significant signs of objects in the image. The feature is that each neuron of this layer is completely connected only to one sign of the previous layer. The last layer is a day off and comprises the number of neurons, equal to quantity of classes.

The value on an output of neuron of a convolution layer is calculated by means of expression

$$
y_{m}^{i, j}=f b_{m}+w_{s=1 t=1 k=1}^{s, t} x^{i-1+s, j-1+t, k}
$$

$$
i=\overline{1, w_{\text {out }}}, j=1, h_{\text {out }}, s, t=1, K
$$

where $y_{m}^{(i, j)}$ - neuron signal m's card of signs of an output layer; $w_{m}^{(s, t)}$ - weights, matrix of synoptic coefficients m's card of signs of an output layer, multi-channel input signal; $K-$ size of the receptive plane (synoptic filter), the threshold, neural offset m's card of signs - reflects increase or reduction of the input signal given on activation function; $k$ - the card number of signs in the input channel (layer); $L$ - total quantity of card of signs in the input channel (layer); $w_{\text {out }}, h_{\text {out }}-$ size of a card of signs of an output layer.

The value on an output of neuron of a subselection layer is calculated by means of expression:

$$
y_{m}^{(i, j)}=\max x^{i+s q_{a}, j+s q_{b}, m} \begin{gathered}
i=1, w_{\text {out }} \\
, j=1, h_{\text {out }} \\
a, b=1,2
\end{gathered}
$$

where $y_{m}^{(i, j)}-$ output signal (neuron) m's card of signs, $s q_{a}=i, i-1, s q_{b}=(j, j-1)-$ vectors with coordinates of elements of the input plane, $x^{(*)}-$ input.

Thus, this approach can classify with a certain accuracy images of microsection of metal for the purpose of prediction of characteristics of material with the certain accuracy depending on quantity of elements of a learning subset and variety of its contents. If the big set of different structures will be provided by many images, the qualifier will work more precisely and vice versa. The positive side of this approach is that in case of the qualitative learning database it is possible to describe rather easy way the qualifier that will have in itself several layers with a static size of the input image in pixels.



Fig. 4. Granular perlite

We will consider formation of a learning set for the decision of the problem of classification by a one-stage method. In this case, the basis for a training of a neuralnetwork will consist of 3500 pictures of microsections of the metal. It is important to mark that the learning set shall contain pictures of various microstructures. The set of physic 
mechanical characteristics of material directly depends on the structure of the metal.

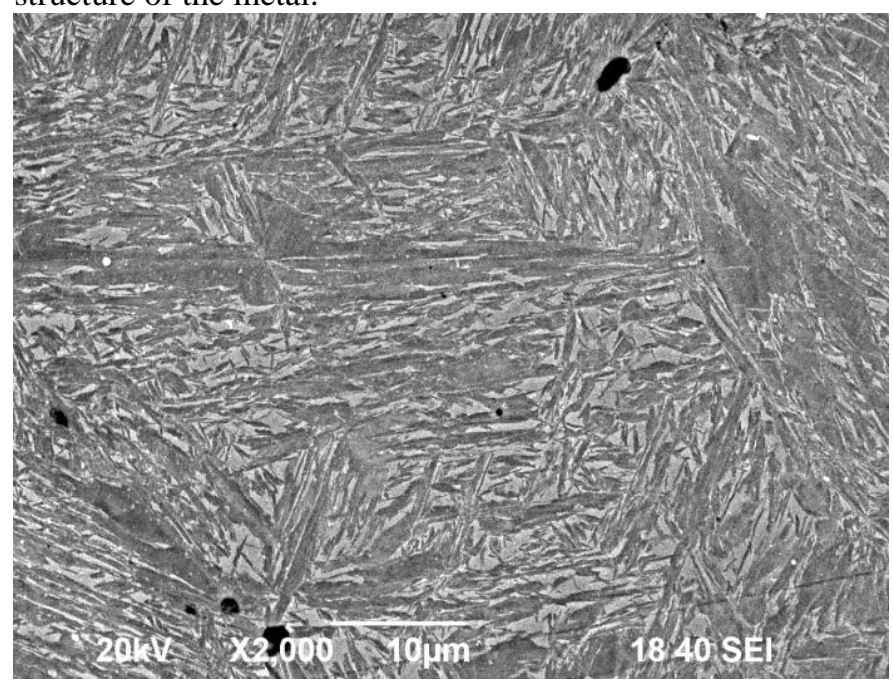

Fig. 5. Martensite

For example, the granular perlite provided in Fig. 4 has microhardness $1900 \mathrm{MPa}$. At the same time, the hardness of the martensite given in Fig. 5 considerably above also is 7000 $\mathrm{MPa}$. The provided pictures are made with a multiplicity of increase in X2000. In case of such increase differences in a microstructure of materials therefore it is important for each considered characteristic of a microstructure to have the appropriate pictures in which differences in, for example, point of grain, interlamellar distance, etc. are well visible.

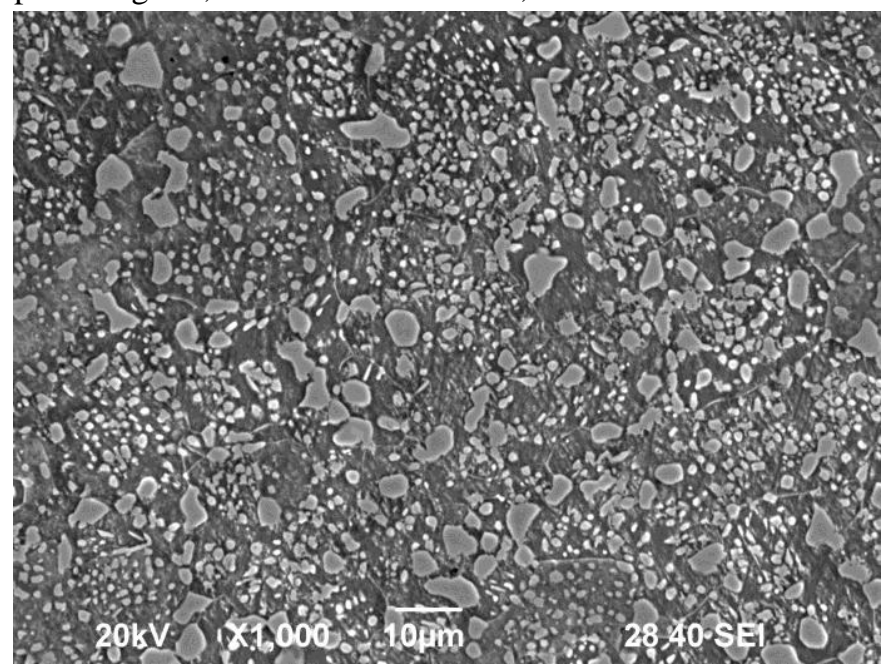

Fig. 6. Granular perlite.

Besides, in a case when basic selection of pictures is insufficiently volume for achievement of required accuracy of classification perhaps extension of a basis in several ways. We will list some of them: reflection across, accidental framing, turn, distortion and others. It is important to mark that augmentations with change of color and accidental scaling in case of the decision of this task are inappropriate in view of the fact that the scale of increase participates as the characteristic of the image for the reasons that a part of properties of metal is perceptible only in case of a certain increase. As an example we will consider pictures of metal, provided in Fig. 4, but already made with increase in X1000 (Fig. 6).

With the naked eye the difference in grains sizes in pictures therefore any transforming augmentations except turn and reflection can distort the end result is visible.

The lack of such approach is shown that by transmission of new functional material which microstructure was not earlier provided in a learning set such qualifier will not be able correctly to recognize structure. In view of the fact that the experimental materials need to be evaluated with a certain accuracy on compliance to the given physical-mechanical properties, the second approach which feature consists in separation of areas of the image and assignment by it of a possibility of influence on a certain row of characteristics is offered. Thus, if new metal consists of new structure which only partially repeats the samples provided in learning selection, the qualifier will alleged more precisely estimate compliance of microsection to the predicted characteristics.

\section{TWO-STAGE APPROACH}

In the other hand, there is other decision method for resolving the problem of classification set which has been demonstrated earlier in this paper. Unlike the described earlier one-stage approach in which the direct problem of classification by transmission to an input of a neural network of the complete image and selection of values of scales of the qualifier is solved, it is possible to try to improve classification accuracy, beforehand having solved the problem of segmentation of the image.

The input data preparation for the approach described earlier as the decision of the problem of classification becomes key feature in this case. From a basis of the materials, images, which are widely applied in technique and separately the functional materials, are selected separately. For establishment of hypothetical dependence between objects on the image and the actual physic mechanical properties it is offered to solve the problem of segmentation. For this purpose, the problem of finding of the general elements on the images responding to the close characteristics is set for a neural network (for example, search of the general objects at different pictures of metals with an identical durability). So, the average interlamellar distance of the material provided in Fig. 7 at the left matters is 0.155 microns while at material on the right 0.144 microns. The provided materials in case of similar distances have values of microhardness of 3016 MPas and 3095 MPas respectively, and it will allow a neuronet to draw a conclusion on the connectivity of existence of indents of a certain size about estimated hardness. It is worth marking that the provided image has coefficient of increase in X40000, and increases the distance. From here, it is possible to draw a conclusion that the segments found in training activity need to be bound to image scale.

After execution of such operation before a neural network there will be a set of templates in case of the decision of the 
problem of classification, namely small sections of the image which will be considered by search of a class of the image. In other words, the qualifier when training will rely not on only communication of the complete image with its characteristics, and on the image connected to a set of areas of interest (ROI) which in turn will be compared to samples. Such pre-training will increase the accuracy of recognition of the image and its classification.

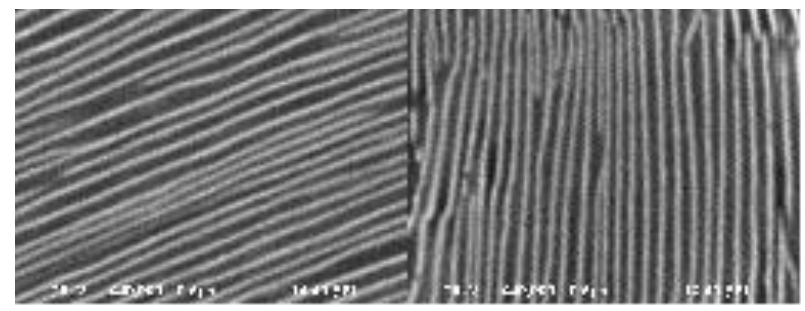

Fig. 7. Example of images of one increase with similar properties

On completion of the decision of the task of segmentation, the problem of classification by means of the algorithm described above is solved. At the same time on an input, not all images of a microstructure but only its parameters or objects significantly influencing the predicted characteristics researched by FM move.

\section{RESULTS AND DISCUSSION}

Two approaches to the decision of the problem of metal microsection classification including a row of stages are offered. In case of the first approach one stage at which the direct problem of the microstructure image picture classification of the figured material researched by FM on the given set of values of physical-mechanical characteristics by means of a neural network is solved and supposed. The second approach consists of two stages, on the first the problem of segmentation of the image is solved, and then on the selected subjects of the image the decision of the problem of microstructure classification is passed. At the same time at each stage, different neuronets are used.

It is important to mark that a learning subset of images for both approaches is different. In the first case, it represents the integral database consisting of all available pictures of microsections with their described characteristics, and in a case with two-stage approach it is offered to divide selection into two databases: the first - widely applied materials, the second - the researched functional metal materials.

It is shown that in case of the decision of the microstructures classification problem by means of the offered approaches it is important to have several pictures of the same material at different scales of increase. It is connected to the fact that some subjects of the image of a microstructure significantly influencing on the physical-mechanical properties researched by FM, can be insufficiently obviously expressed at certain scales of increase and it will reduce the accuracy of the decision of the problem of classification.

\section{Acknowledgment}

The research was conducted with financial support of the Ministry of Education and Science of the Russian Federation within the RFMEFI58617X0055 project. The name of a joint project with the EU within the Horizon of 2020 program: "Fracture across Scales and Materials, Processes and Disciplines" project Acronym: "FRAMED".

\section{References}

[1] A. Arlazarov at al. "Evolution of microstructure and mechanical properties of medium Mn steels during double annealing.", Materials Science and Engineering A 542 (2012) 31-39 pp, April 2012.

[2] C.S. Kim, "Variation of mechanical characteristics and microstructural evolution in AISI 316 austenitic stainless steel subjected to long-term thermal aging at elevated temperature.", Strength of Materials, Vol. 49, No. 2, March, 2017.

[3] A.V. Klyuev, V.Yu. Stolbov, S.I. Sharybin, "Visualization of the Complex Grain Structure of Metals and Alloys in their Parameters Identification”, Scentific Visualization. Vol. 8, No 3. pp. 95-101, 2016.

[4] Waseem Khan, "Image Segmentation Techniques: A Survey", Journal of Image and Graphics, Vol. 1, No. 4, pp. 166-170, 2013.

[5] M. Sharma, S. Singh, "Evaluation of texture methods for image analysis", The Seventh Australian and New Zealand Intelligent Information Systems Conference, 2001, pp. 117-121, 18-21 Nov. 2001.

[6] S. Rassel, P. Norving, Isskustvenniy intellect: sovremenniy podhod, M.: Izdat.dom «Wiliams», 2006, p. 1408.

[7] L.N. Yasnitskiy, Intellectualnie systemy, M.: Laboratoria znaniy, 2016, p. 221.

[8] Large Scale Visual Recognition Challenge 2012 (ILSVRC2012) Retrieved from: http://imagenet.org/challenges/LSVRC/2012/results.html

[9] Y. LeCun, A theoretical framework for backpropagation, Proceedings of the 1988 Connectionist Models Summer School, pp.21-28 CMU, Pittsburgh, Pa 1988 .

[10] Y. LeCun, L. Bottou, Y. Bengio, P. Haffne ,"Gradient-Based Learning Applied to Document Recognition”, Proc. IEEE, pp.59-67, 1998.

[11] Zhou Shiyang, Chen Youping, Zhang Dailin, Xie Jingming, Zhou Yunfei, "Classification of surface defects on steel sheet using convolutional neural networks", Materiali in tehnologije, Vol. 51, pp. $123,2017$.

[12] J. Masci, U. Meier, D. Ciresan, J. Schmidhuber, G. Fricout, "Steel defect classification with Max-Pooling Convolutional Neural Networks", The 2012 International Joint Conference on Neural Networks (IJCNN), pp. 6-131, 2012. 